\title{
Nature and severity of lung function abnormalities in extremely pre-term children at 11 years of age
}

\author{
S. Lum*, J. Kirkby*, L. Welsh*, N. Marlow", ${ }^{*}$, E. Hennessy ${ }^{+}$and J. Stocks*
}

ABSTRACT: Advances in neonatal care have resulted in increased survival of children born extremely pre-term (EP). Nevertheless the incidence of bronchopulmonary dysplasia and longterm respiratory morbidity remains high. We investigated the nature of pathophysiological changes at 11 yrs of age to ascertain whether respiratory morbidity in EP children primarily reflects alterations in the lung periphery or more centralised airway function in this population.

Spirometry, plethysmography, diffusing capacity, exhaled nitric oxide, multiple-breath washout, skin tests and methacholine challenge were used during laboratory-based assessments in a subgroup of the 1995 EPICure cohort and in controls.

Results were obtained in 49 EP and 52 control children. Lung function abnormalities were found in $\mathbf{7 8 \%}$ of EP children, with evidence of airway obstruction, ventilation inhomogeneity, gas trapping and airway hyperresponsiveness. Levels of atopy and exhaled nitric oxide were similar between the groups. Prior wheeze was associated with significant reductions in forced flows and volumes. By contrast, abnormalities of the lung periphery appear to be mediated primarily through EP birth per se.

The prevalence of lung function abnormalities, which is largely obstructive in nature and likely to have long-term implications, remains high among 11-yr-old children born EP. Spirometry proved an effective means of detecting these persistent abnormalities.

KEYWORDS: Bronchopulmonary dysplasia, child, extreme prematurity, respiratory follow-up

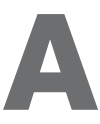
dvances in neonatal care over the past few decades have resulted in increasing survival of babies born less than 25 completed weeks of gestation (extremely pre-term (EP)) [1]. Nevertheless, the prevalence of bronchopulmonary dysplasia (BPD), remains high in such infants [2], and our understanding of the implications of $\mathrm{EP}$ birth and any subsequent lung injury on lifelong lung function (LF) remains limited.

A diagnosis of BPD was initially limited to early recipients of neonatal mechanical ventilation; the associated pulmonary pathology being primarily attributed to iatrogenic damage from barotrauma and oxygen toxicity during the late saccular-early alveolar phase of lung development, with functional abnormalities persisting to adulthood [2]. Computerised tomography has revealed a high incidence of structural alterations in these early survivors of BPD [3]. By contrast, since widespread introduction of antenatal corticosteroids and neonatal surfactant therapy in the early 1990s, $\mathrm{BPD}$ is now largely restricted to more immature infants delivered during the early saccular phase and "new" BPD has been reported to be characterised by disruption of alveolar development, with reduced alveolar number and enlarged airspaces, but less pulmonary fibrosis and lung injury than previously described [4]. Nevertheless, the degree of persistent airway obstruction, as reflected by spirometry, has remained remarkably constant $[2,5,6]$. Given that diminished forced expiratory volume in $1 \mathrm{~s}$ (FEV1) is a marker of all-cause premature mortality [7] and that those with low LF at initial assessment tend to remain low at subsequent assessments and vice versa [8], there is concern that survivors of preterm birth may be at risk of early onset chronic obstructive pulmonary disease in adulthood [3].

Although a wide range of tests have been used to assess cardio-respiratory function in survivors of

This article has supplementary material available from www.erj.ersjournals.com

A press release for this article is available from www.erj.ersjournals.com/site/misc/presspack.xhtml

AFFILIATIONS

*Portex Unit, Respiratory Physiology and Medicine, UCL, Institute of Child Health,

'Institute of Women's Health, UCL, +Wolfson Institute, Barts and the London School of Medicine and Dentistry, Queen Mary University of London, London, and \#School of Human Development, University of Nottingham, Nottingham, UK.

CORRESPONDENCE

S. Lum

Portex Unit

Respiratory Physiology and Medicine UCL

Institute of Child Health 30 Guilford Street London WC1N 1EH UK E-mail: s.lum@ich.ucl.ac.uk

Received:

May 062010

Accepted after revision: Sept 012010 First published online: Oct 142010 
pre-term birth $[2,5,6,9,10]$, relatively few studies have included the full spectrum of available techniques and spirometric parameters remain the most common outcomes, as used during recent school assessments of survivors of the EPICure cohort at 11 yrs of age [11]. In that study, $56 \%$ of children born before $25 \mathrm{w}^{+6}$ gestation were found to have abnormal baseline spirometry, $27 \%$ had a positive bronchodilator response and $25 \%$ a diagnosis of asthma (twice that observed in classmates). Apart from BPD, which had a detrimental impact on all spirometric outcomes, and duration of post-natal steroids, none of the perinatal or maternal factors were associated with spirometric lung function at $11 \mathrm{yrs}$ of age [11]. However, if the underlying pathophysiology of new BPD primarily reflects peripheral lung disease, a substantial proportion of functional abnormalities could potentially go undetected in EP children, if assessments are limited to spirometry $[12,13]$.

The aim of our study was to ascertain whether ongoing respiratory morbidity in EP children born in the 1990s primarily reflects alterations in the lung periphery, as assessed using multiple breath inert gas washout, plethysmography (partitioned lung volumes) and diffusion capacity or more centralised airway function (spirometry and airway resistance), and whether there was any evidence of increased eosinophilic airway inflammation (i.e. elevated levels of exhaled nitric oxide fraction (FeNO) in such children). We hypothesised that: 1) LF would be diminished at 11 yrs of age in children born EP when compared with full-term controls; 2) use of spirometry alone would underestimate the true degree of morbidity; and 3) changes in LF would not be accompanied by increased airway inflammation. None of the results presented in this study have been reported previously, except as abstracts.

\section{METHODS}

EPICure is a population-based study of all births at $\leqslant 25^{+6}$ weeks of gestation in the UK and Republic of Ireland between March and December 1995 [11, 14]. The laboratory-based investigation described below was part of an extensive assessment performed at 11 yrs of age, which included spirometric measurements in the entire school cohort [11]. An age, sex and ethnic-group matched classmate for each EP child was sought. Classmates were excluded if they had been born at $<37$ weeks gestational age, had been previously hospitalised for a respiratory complaint or had suffered from $\mathrm{TB}$, pneumonia or whooping cough. Asthma and atopy were not exclusion criteria. Asthma classification was based on parental report of doctordiagnosed asthma. Our operational definition of "current asthma" was use of asthma medication or wheeze in the past 12 months by children with doctor-diagnosed asthma or use of asthma medication and wheeze in the past 12 months even if no prior diagnosis of asthma had been reported.

Index and control children living within reasonable travelling distance of London (UK) were recruited for extensive respiratory assessments at the UCL, Institute of Child Health (ICH; London, UK) (fig. 1). The study was approved by the ICH Research Ethics Committee. Parental written consent and assent from each child were obtained.

Lung function tests (LFTs) were performed according to American Thoracic Society/European Respiratory Society standards with investigators blinded to birth status. Assessments

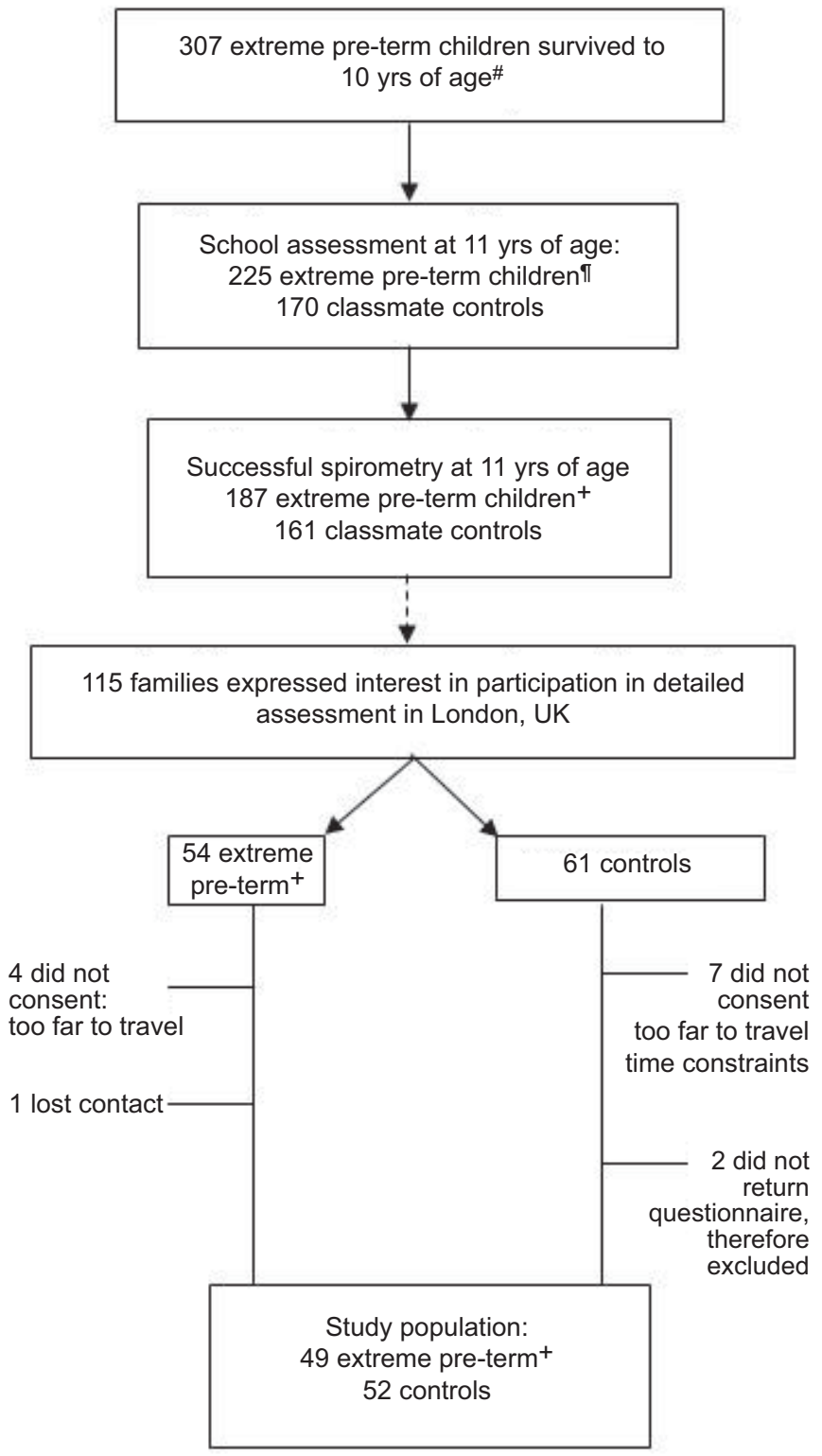

FIGURE 1. Study population: subject recruitment and accrual. *: includes one child not identified in 1995, but eligible to be in the 1995 EPICure cohort; " : includes six children born in January 1996 who were recruited to the EPICure study but not included in the cohort analyses, as they were born after December 31, 1995;

+: including two out of the six children in born in January 1996.

included spirometry, plethysmography, diffusing capacity of the lung for carbon monoxide, FeNO, multiple-breath inert-gas washout to assess ventilation inhomogeneity, skin allergy test and methacholine challenge (refer to supplementary data). Doctor-diagnosed asthma, medication use and current respiratory symptoms including wheeze were determined by parental response to the International Study of Asthma and Allergies in Childhood (ISAAC) questionnaire. Each child was requested to rate his/her own pubertal status [15]. This extensive study protocol was performed in two laboratory sessions within a 3week interval. 


\section{Power of study}

A sample size of 50 children in each group would provide at least $80 \%$ power to detect differences in lung function $>0.57 \mathrm{sD}$ between index and control groups, at the 5\% significance level.

\section{Data management and statistical analysis}

Codes regarding birth status were not released until lung function data collection and analysis had been completed. Neonatal data [16] were used to identify the EP children who had had BPD, defined as those still receiving supplemental oxygen at 36 weeks post-menstrual age [2].

With the exception of the Lung Clearance Index (LCI), which, in health, is relatively constant throughout life [12], lung function results were expressed as Z-scores to adjust for height, sex and age [17-20]. Results were classified as: normal when total lung capacity (TLC), forced vital capacity (FVC), FEV1 and forced expiratory flow at $25-75 \%$ of FVC (FEF25-75\%) all fell within the normal range; obstructive, when FEV1/FVC was less than the lower limit of normal (LLN) and/or residual volume (RV)/TLC was greater than upper limit of normal; or restrictive when TLC and/or FVC were less than LLN or FEV1 and/or $\mathrm{FEF} 25-75 \%$ was reduced in the presence of a normal FEV1/FVC [21]. The extent to which extreme prematurity (EP) and respiratory morbidity were associated with lung function at 11 yrs of age was examined using multiple linear regression (MLR) (SPSS version 15.0; SPSS, Chicago, IL, USA). Data management was undertaken using Re-Base software (J7 Group, Rickmansworth, UK).

\section{RESULTS}

\section{Population characteristics}

Successful lung function assessments and completed questionnaires were obtained from $49 \mathrm{EP}$ and 52 classroom controls (fig. 1), recruited from 20 out of 39 counties in England, UK. No potential controls had to be excluded due to prior hospitalisation or serious respiratory morbidity (see exclusion criteria). Table 1 summarises group characteristics according to birth status.

\section{How representative was the study population?}

With the exception of exposure to maternal smoking during pregnancy, which was higher among children who were only tested in school, the subgroup of EP children attending the extended respiratory assessments at $\mathrm{ICH}$ was representative of the entire cohort tested in school with respect to neonatal and maternal characteristics, neonatal history, respiratory morbidity and spirometry (table 2). Similarly there was no significant difference between the control group assessed in the laboratory and those assessed at school (data not shown). The ethnic mix of the subgroup of EP children attending laboratory testing was representative of those tested at school. However, although classroom controls were well matched for ethnic group with the EP children during school assessments of the entire cohort [15], slightly fewer non-White controls attended the laboratory assessments (table 1).

At time of the test, EP children were shorter and lighter than controls (table 3). After adjustment for prematurity, age at test was similar between EP children and controls (10.9 versus 11.0 yrs respectively), while anthropometric differences remained highly

TABLE 1 Group characteristics of extreme pre-term (EP) children with and without prior bronchopulmonary dysplasia (BPD) compared to classmate controls

\begin{tabular}{|c|c|c|c|c|c|}
\hline & EP with BPD & EP without BPD & All EP & Control & $\begin{array}{c}95 \% \mathrm{Cl} \text { of difference } \\
\text { (EP-control) }\end{array}$ \\
\hline \multicolumn{6}{|l|}{ Neonatal characteristics } \\
\hline Subjects $n$ & 34 & 15 & 49 & 52 & \\
\hline Boys & 35 & 40 & 37 & 37 & $-18-18$ \\
\hline Gestational age weeks & $24.8 \pm 0.8$ & $25.0 \pm 0.6$ & $24.9 \pm 0.7$ & $40.1 \pm 1.6$ & $-15.7-14.7^{\star \star \star}$ \\
\hline Birth weight kg & $0.740 \pm 0.101$ & $0.762 \pm 0.093$ & $0.747 \pm 0.098$ & $3.361 \pm 0.450$ & $-2.75-2.48^{\star \star *}$ \\
\hline Birth weight Z-score ${ }^{\#}$ & $-0.06 \pm 0.71$ & $-0.03 \pm 0.80$ & $-0.05 \pm 0.74$ & $-0.24 \pm 1.14$ & $-0.57-0.20$ \\
\hline BPD & 100 & 0 & 69 & & \\
\hline Post-natal steroids & 70 & 67 & 69 & & \\
\hline \multicolumn{6}{|l|}{ Maternal/family details } \\
\hline Antenatal steroids & 85 & 87 & 86 & & \\
\hline PROM & 15 & 31 & 19 & & \\
\hline Chorioamnionitis & 21 & 39 & 26 & & \\
\hline Smoking in pregnancy & 25 & 14 & 22 & 22 & $-16-17$ \\
\hline Current smoking exposure & 18 & 7 & 15 & 26 & $-27-4$ \\
\hline Maternal asthma & 12 & 0 & 8 & 12 & $-16-9$ \\
\hline $\mathrm{FH}$ of asthma & 38 & 33 & 37 & 33 & $-14-22$ \\
\hline Maternal ethnicity White & 74 & 87 & 78 & 94 & $-30-3^{*}$ \\
\hline Maternal education after age 16 yrs & 52 & 57 & 53 & 73 & $-38-0^{*}$ \\
\hline Non-manual occupation & 74 & 79 & 76 & 69 & $-10-29$ \\
\hline
\end{tabular}

Data presented as $\%$ or mean \pm SD, unless otherwise indicated. BPD is defined as oxygen given at or beyond 36 weeks post-menstrual age. PROM: prolonged rupture of membranes ( $>24$ h). *: $p<0.05 ;{ }^{\star *}: \mathrm{p}<0.01 ;{ }^{* \star *}: \mathrm{p}<0.0001 .{ }^{*}$ : according to Child Growth Foundation algorithms [22]; ${ }^{\circledR}$ : classification based on either parent with a nonmanual occupation. 


\begin{tabular}{|c|c|c|c|c|}
\hline \multirow{3}{*}{\multicolumn{2}{|c|}{ TABLE 2}} & \multicolumn{3}{|c|}{ espiratory laboratory at the Institute of Child Health $(\mathrm{ICH}$; } \\
\hline & & \multicolumn{2}{|c|}{ EP } & \multirow[t]{2}{*}{$95 \% \mathrm{Cl}$ of difference (school- $\mathrm{ICH}$ ) } \\
\hline & & School tested & $\mathrm{ICH}$ tested & \\
\hline \multicolumn{2}{|c|}{ Subjects (\% males) } & $140(44)$ & $49(37)$ & $-9-22$ \\
\hline \multicolumn{2}{|c|}{ Birth weight Z-score $\#$} & $-0.17 \pm 0.78$ & $-0.05 \pm 0.73$ & $-0.36-0.13$ \\
\hline \multicolumn{2}{|l|}{ BPD } & 71 & 69 & $-12-16$ \\
\hline \multicolumn{2}{|c|}{ Received ANS } & 80 & 86 & $-16-8$ \\
\hline \multicolumn{2}{|c|}{ History of chorioamnionitis } & 22 & 26 & $-18-10$ \\
\hline \multicolumn{2}{|c|}{ Wheeze in last 12 months } & 23 & 22 & $-13-14$ \\
\hline \multicolumn{2}{|c|}{ FEF $25-75 \%$ Z-score ${ }^{+}$} & $-2.0 \pm 1.3$ & $-2.0 \pm 1.2$ & $-0.4-0.4$ \\
\hline
\end{tabular}

Data presented as mean \pm SD or \%, unless otherwise stated. BPD: bronchopulmonary dysplasia, defined as oxygen given at or beyond 36 weeks post-menstrual age; ANS: antenatal steroid; FEV1: forced expiratory volume in $1 \mathrm{~s}$; FEF25-75\%: forced expiratory flow at 25-75\% forced vital capacity. Comparison of FEV1 and FEF25-75\% Z-scores was undertaken by using data obtained from both groups during school assessments. *: $p<0.05$. ${ }^{*}$ : according to [22]; ": defined as currently symptomatic and/ or with doctor-diagnosis of asthma and on medication during past 12 months; ${ }^{+}$: according to [20].

significant. A similar proportion of EP and controls had reached the onset of puberty at the time of the test.

Prior respiratory morbidity (table 3) was significantly more common in the EP children and both current and inactive asthma were almost twice as frequent than in controls, but there was less difference in atopic status (eczema and/or positive skin test) or prevalence of hayfever (table 3). In the 12 months prior to testing, significantly more EP children with prior BPD were symptomatic (i.e. wheezed or had been treated for respiratory illness), although only one child was receiving antibiotics at testing. Incidence of wheeze in the 3-month period preceding LFTs (table 4) was generally similar in EP children without prior BPD to that in controls, except when associated with a cold. In contrast, prior BPD was associated with significantly more recent wheeze, irrespective of how it was categorised (table 4 ). Wheeze with colds was associated with EP status (OR 8.1, 95\% CI 1.7-38.4; $\mathrm{p}=0.008$ ) and among EP children, prior BPD was associated with shortness of breath during exercise (OR 5.7, 1.4-24.1; $\mathrm{p}=0.017$ ).

\section{LF results}

Effect of extreme prematurity

With the exception of static lung volumes (functional residual capacity, TLC and alveolar volume), significant impairments in all LF variables were found among EP children when compared with controls (table 5, fig. 2), with evidence of airway obstruction (decreased forced expiratory flows and volumes, elevated specific airways resistance), ventilation inhomogeneity, gas trapping (elevated RV/TLC) and airway hyperresponsiveness abnormalities being most marked in EP children with BPD. By contrast, levels of FeNO and incidence of atopy were similar between EP and control children. Details of univariable analysis are presented in table E1 in the supplementary data.
Associations between airway function, extreme prematurity and respiratory morbidity

On multivariable analysis, after adjusting for ethnicity, being born EP was significantly associated with decrements in all LF outcomes, while respiratory morbidity (wheeze ever) was associated with further decrements of central airway function (table 6), but not with markers of more peripheral lung function such as LCI (coefficient 0.2, 95\% CI -0.1-0.6; $\mathrm{p}=0.19$ ), gas trapping (RV/TLC $0.4,95 \%$ CI $-0.05-0.8 ; \mathrm{p}=0.08)$ or diffusing capacity $(0.1,95 \%$ CI $-0.3-0.5 ; \mathrm{p}=0.69)$.

\section{Extent to which spirometry identified respiratory abnormalities in EP children}

In the EP children, 11 (22\%) had no LF abnormalities (although two of these had incomplete tests) and 38 had some LF abnormalities. Spirometry identified $24(63 \%)$ EP children with LF abnormalities, LCI 20 (58\%) EP children and sReff 17 (45\%) EP children, while a combination of spirometry and sReff identified 30 (79\%) out of the $49 \mathrm{EP}$ children with abnormalities.

Of the 47 out of $49 \mathrm{EP}$ children with acceptable spirometry and plethysmography, 21 (45\%) exhibited an obstructive pattern, of whom $14(67 \%)$ had a history of wheeze and $10(48 \%)$ a diagnosis of asthma, while only five (11\%) had evidence of restrictive lung disease on spirometric criteria (all of whom had a history of wheeze, but only one a diagnosis of asthma). Of these five, technically acceptable results of partitioned lung volumes were only available from two, of whom one had a reduced TLC.

\section{DISCUSSION}

This study represents the most extensive respiratory assessments in children of such low gestation to date, and reveals a wide spectrum of functional abnormalities in both the larger airways and lung periphery at $11 \mathrm{yrs}$ of age in children 
TABLE 3 Group characteristics of extreme pre-term (EP) children with and without prior bronchopulmonary dysplasia (BPD) compared to classmate controls at $11 \mathrm{yrs}$ of age

$$
\begin{array}{llll}
\text { EP with BPD } & \text { EP without BPD } & \text { All EP } & \text { Control }
\end{array}
$$

(EP-control)

\begin{tabular}{|c|c|c|c|c|c|}
\hline Subjects (\% boys) & $34(35)$ & $15(40)$ & $49(37)$ & $52(37)$ & $-18-18$ \\
\hline Height $Z^{\#}$ & $-0.47 \pm 0.92$ & $-0.29 \pm 1.08$ & $-0.41 \pm 0.97$ & $0.35 \pm 0.96$ & $-1.14-0.38^{\star \star \star}$ \\
\hline Weight $Z^{\#}$ & $-0.34 \pm 1.19$ & $-0.05 \pm 1.42$ & $-0.25 \pm 1.26$ & $0.43 \pm 1.05$ & $-1.14-0.22^{\star \star}$ \\
\hline BMI Z & $-0.13 \pm 1.33$ & $0.27 \pm 1.12$ & $-0.01 \pm 1.27$ & $0.35 \pm 1.08$ & $-0.82-0.11$ \\
\hline Puberty & 29 & 29 & 29 & 29 & $-18-18$ \\
\hline Pneumonia $^{+}$ & 19 & 9 & 17 & & \\
\hline Wheeze ever & 62 & 27 & 51 & 21 & $12-48^{\star \star}$ \\
\hline Wheeze last 12 months $^{\S}$ & 29 & 7 & 23 & 10 & $-2-27$ \\
\hline Inactive asthma ${ }^{f}$ & 15 & 13 & 14 & 8 & $-6-20$ \\
\hline Current asthma $a^{\# \#}$ & 29 & 20 & 27 & 13 & $-3-28$ \\
\hline$\beta$-agonist & $9(26)$ & $3(20)$ & $12(25)$ & $5(10)$ & $0-30^{*}$ \\
\hline Inhaled steroids & $9(26)$ & $2(13)$ & $11(22)$ & $6(12)$ & $-4-26$ \\
\hline Antibiotics & $8(24)$ & $1(7)$ & $9(18)$ & $2(4)$ & $2-28^{*}$ \\
\hline \multicolumn{6}{|l|}{ Current medication } \\
\hline$\beta$-agonist & $8(24)$ & $1(7)$ & $9(18)$ & $4(8)$ & $-3-24$ \\
\hline Inhaled steroids" & $3(9)$ & $2(13)$ & $5(10)$ & $4(8)$ & $-9-15$ \\
\hline
\end{tabular}

born EP. After adjusting for prematurity, prior wheeze was the strongest predictor for decrements in spirometric airway function, while current asthma was associated with significant increases in airways resistance. Had assessments been limited to spirometry, $37 \%$ of LF abnormalities would have gone undetected. Nevertheless, spirometry remained the most discriminative test in this population, as well as being the most feasible for use in field studies.

\section{Strengths and limitations}

Strengths of this study include the fact that the EP subset who attended laboratory tests were representative of the national cohort [11]. As measurements were limited to children who were sufficiently coordinated to perform the extensive range of laboratory-based tests, findings may underestimate the true extent of lung dysfunction. Investigators were blinded to birth status, and recruitment of a prospective control group that was representative of the local population, including incidence of asthma [23], allowed us to assess the effects of EP birth over and above that of asthma [11]. By categorising functional abnormalities based on limits of normality derived from local healthy controls (supplementary table E2), we avoided potential errors that may occur when relying simply on published reference data (www.growinglungs.org.uk) [6, 24]. A reduction in spirometric parameters among Black and Asian subjects when compared to those of White European descent is well recognised [24], and was confirmed in this study, but differences between groups remained similar whether or not the relatively few non-White children were included in the analyses.

The extensive nature of laboratory assessments, which also included exercise [14] and neuro-sensory testing [25], and the need to travel to central London, limited the sample size and hence the power with which subgroup analyses could be reliably undertaken. Nevertheless, the trends towards increased morbidity and functional deficits in those with prior BPD who participated in the laboratory assessments mirrored the significant changes found in the entire cohort [11]. As reported previously [11], maternal smoking in pregnancy was not associated with changes in any LF outcome, with the EP children possibly having been delivered before the anticipated adverse effects occurred. We could not examine the potential impact of intrauterine growth retardation in this population [10] due to extremely low survival of such infants at these gestations. 


$\begin{array}{lccc}\text { TABLE } 4 & \begin{array}{l}\text { Pattern of wheeze in the past } 3 \text { months } \\ \text { EP with BPD }\end{array} & \begin{array}{l}\text { EP without BPD } \\ \text { Control }\end{array} & \\ & & 15 & 34 \\ \text { Subjects } \mathbf{n} & 34 & 7 & 8 \\ \text { Wheeze during the day } & 24 & 13 & 4 \\ \text { Wheeze with colds } & 29^{\star *} & 7 & 6 \\ \text { Wheeze without colds } & 15 & 7 & 6 \\ \text { Wheeze with exercise } & 26^{*} & 20 & 21^{\#} \\ \text { SOB with exercise } & 59^{\star *} & & \end{array}$

Data are presented as \%, unless otherwise stated. EP: extreme pre-term; BDP: bronchopulmonary dysplasia; SOB: shortness of breath. Exact test for comparison between the three subgroups: ${ }^{*}: p<0.05$; ${ }^{*}: p<0.01$. ${ }^{\#}$ : objective assessments of physical activity showed that all children undertook considerably less physical activity than current recommendations of at least $60 \mathrm{~min}$ of moderate-vigorous physical activity per day which may explain why $21 \%$ of controls experience SOB with exercise.

\section{Respiratory morbidity}

While $29 \%$ of children with prior BPD had doctor-diagnosed asthma and recent asthma medication, there was no increase in either the prevalence of atopy or levels of FeNO in these children, suggesting a different underlying pathophysiology to that usually observed in childhood asthma [26]. The fact that the airways obstruction observed in these children was only partially reversible raises the issue as to whether these children have been correctly diagnosed, or optimally treated [11]. Consistent with previous reports [9, 10], airway responsiveness was increased among the EP children, although had we not undertaken identical challenges in prospective controls the extent of such hyperresponsiveness would have been overestimated (refer to supplementary data). Children with BPD are known to be at increased risk for symptomatic respiratory illnesses in infancy and childhood. This may largely reflect the effects of diminished airway calibre, as indicated by the increased sReff and decreased expiratory flows and volumes found in this population, such that wheeze can be invoked with minimal further airway narrowing. However, neonatal hyperoxia may also exacerbate inflammatory responses, leading to long-term disruption of key innate immuno-regulatory pathways in such subjects [27].

\section{Nature of underlying pathophysiology}

Initial descriptions of new BPD pathology suggested that EP delivery could result in an "arrest" of alveolar development [2] but later reviews suggest impaired alveolisation [4], since there is evidence of continued post-natal alveolar formation despite early lung insults [28]. The reduced gas mixing efficiency (elevated LCI) observed in EP children may reflect some parenchymal or small airway changes, secondary to disruption of the interstitial collagen network that has been reported following neonatal positive pressure ventilation [29]. While the LCI has been shown to be an early indicator of airway disease

TABLE 5 Lung function results in extreme pre-term (EP) children compared to classmate controls at 11 yrs of age

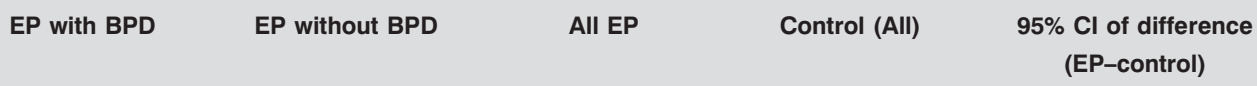

\begin{tabular}{|c|c|c|c|c|c|}
\hline $\mathrm{zFEV}_{1}{ }^{\#}$ & $-1.76 \pm 1.02$ & $-1.08 \pm 1.29$ & $-1.55 \pm 1.14$ & $-0.02 \pm 0.90$ & $-1.94-1.10^{* * *}$ \\
\hline zFEF25-75\% ${ }^{\#}$ & $-2.18 \pm 1.07$ & $-1.55 \pm 1.38$ & $-1.98 \pm 1.19$ & $-0.59 \pm 1.02$ & $-1.85-0.94^{* * *}$ \\
\hline $\mathrm{zFVC}^{\#}$ & $-1.02 \pm 0.85$ & $-0.43 \pm 1.26$ & $-0.84 \pm 1.02$ & $0.22 \pm 0.97$ & $-1.46-0.65^{\star * *}$ \\
\hline $\mathrm{zFEV}_{1} / \mathrm{FVC}^{\#}$ & $-1.27 \pm 0.99$ & $-0.98 \pm 1.23$ & $-1.17 \pm 1.07$ & $-0.40 \pm 0.91$ & $-1.18-0.37^{* * *}$ \\
\hline $\mathrm{zRV}^{*}$ & $1.84 \pm 1.30$ & $1.25 \pm 0.57$ & $1.63 \pm 1.13$ & $1.17 \pm 0.71$ & $0.05-0.88^{*}$ \\
\hline zTLC" & $0.16 \pm 0.41$ & $0.41 \pm 0.57$ & $0.25 \pm 0.66$ & $0.31 \pm 0.56$ & $-0.29-0.17$ \\
\hline 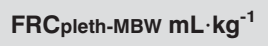 & $12.7 \pm 8.8$ & $12.0 \pm 6.3$ & $12.4 \pm 7.9$ & $8.5 \pm 5.3$ & $0.8-7.1^{*}$ \\
\hline ZsReff $^{\S}$ & $1.11 \pm 0.91$ & $0.70 \pm 0.76$ & $0.98 \pm 0.88$ & $0.42 \pm 0.73$ & $0.24-0.88^{\star *}$ \\
\hline LCI & $7.4 \pm 1.0$ & $7.1 \pm 0.7$ & $7.3 \pm 0.9$ & $6.5 \pm 0.4$ & $0.46-1.10^{\star \star \star *}$ \\
\hline zDL,Co" & $-1.08 \pm 0.90$ & $-1.40 \pm 0.91$ & $-1.20 \pm 0.91$ & $-0.42 \pm 0.91$ & $-1.19-0.37^{*}$ \\
\hline$z V_{A}^{*}$ & $1.28 \pm 1.24$ & $1.36 \pm 0.68$ & $1.31 \pm 1.05$ & $1.41 \pm 1.17$ & $-0.60-0.41$ \\
\hline $\mathrm{zDL}, \mathrm{CO} / \mathrm{VA}^{\bullet}$ & $-2.15 \pm 0.80$ & $-2.58 \pm 0.67$ & $-2.31 \pm 0.77$ & $-1.69 \pm 0.78$ & $-0.97-0.27^{\star *}$ \\
\hline
\end{tabular}

Data expressed as mean \pm SD Z-scores, unless otherwise stated. Data for provocative dose causing a $20 \%$ fall in forced expiratory volume in $1 \mathrm{~s}$ (FEV 1 ) (PC20) and exhaled nitric oxide fraction ( $F$ eNO) are presented as geometric mean \pm SD and $95 \% \mathrm{Cl}$ of the geometric mean were calculated from the $\mathrm{Log}_{\mathrm{e}}$ of PC20 and exhaled $\mathrm{NO}$. BPD: bronchopulmonary dysplasia; FEF25-75\%: forced expiratory flow between 25-75\% forced vital capacity (FVC); RV: residual volume; TLC: total lung capacity; FRCpleth: plethysmographic functional residual capacity (FRC); FRCpleth-MBW: difference in FRC measured using plethysmography and multiple breath washout; sReff: specific effective airway resistance; $\mathrm{LCl}$ : lung clearance index; $\mathrm{DL}, \mathrm{CO}$ : diffusing capacity of the lung for carbon monoxide; $\mathrm{VA}$ : alveolar volume. 95\% Cl of difference in bold

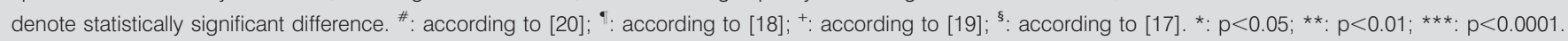



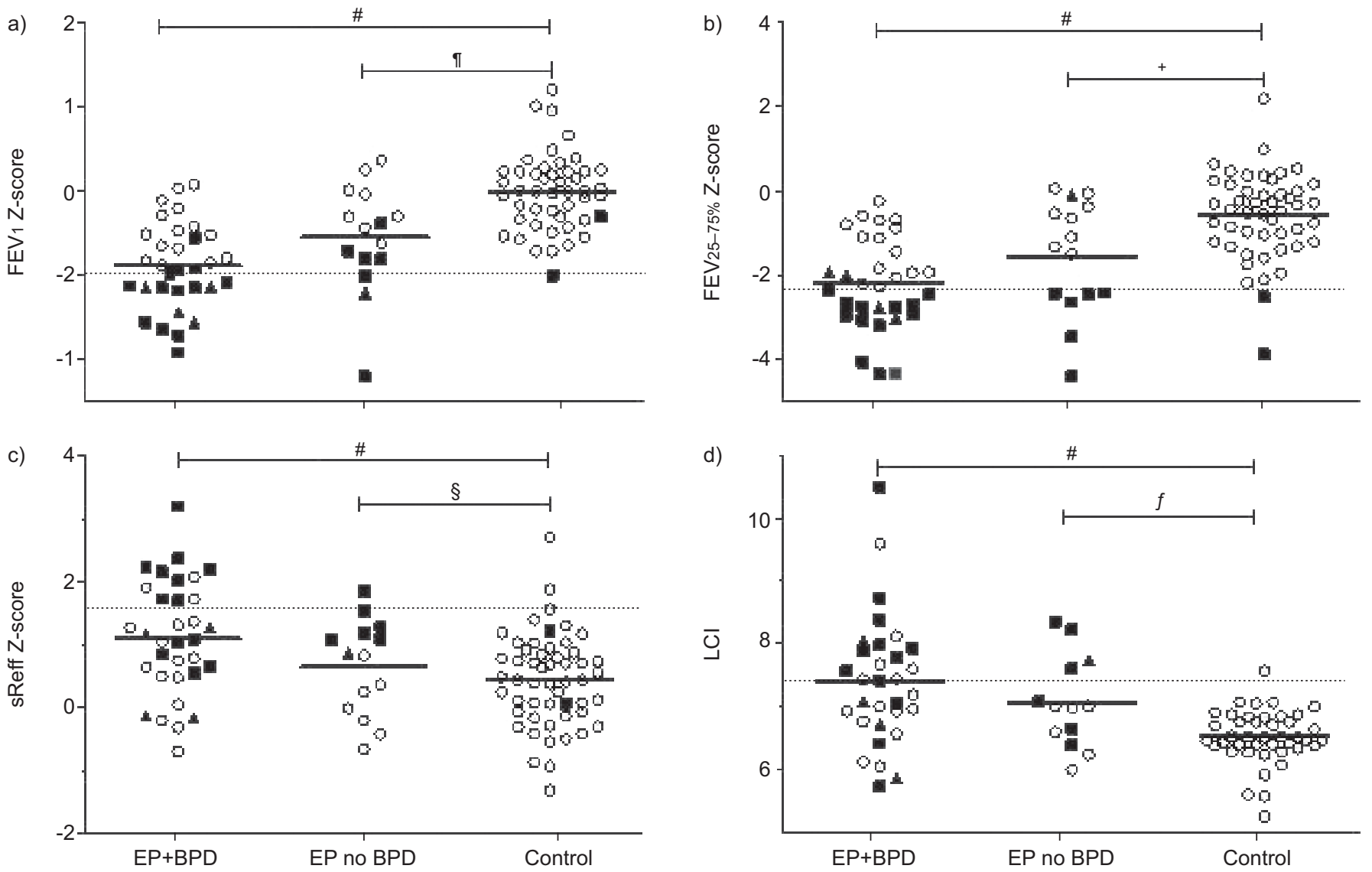

FIGURE 2. Comparison of lung function in children born extremely pre-term with or without bronchopulmonary dysplasia (BPD) and full-term controls according to lung disease categories. —_: mean value for that group. $\mathbf{\square}$ : children classified as having obstructive airways disease; $\mathbf{\Lambda}$ : children classified with restrictive lung disease. Evidence of airway obstruction was only evident in one control with a history of asthma, whose z-score forced expiratory flow between 25-75\% forced vital capacity (FEF25-75\%) was -3.9. The remaining control with forced expiratory volume in $1 \mathrm{~s}(\mathrm{FEV} 1)$ and FEF25-75\% just below lower limit of normal LLN was not asthmatic but had had a lower respiratory tract infection requiring medication in the last 3 months. Unfortunately, technically acceptable lung clearance index (LCl) results were not obtained in either of these controls. sReff: specific effective airway resistance. $\bigcirc$ : no evidence of obstructive or restrictive airway diseases. $\left.{ }^{\#}: p<0.0001 ;{ }^{\natural}: p=0.003 ;{ }^{+}: p=0.004 ;{ }^{\varsigma}: p=0.18 ;{ }^{f}: p=0.02 . a, b\right) \cdots \cdots .:$ lower limit of normal; c, d) …..: upper limit of normal.

in persons with cystic fibrosis [30] it has not been found to be particularly discriminative when assessing BPD or prematurity either during infancy [31,35] or in the current study. A simplified bronchial tree with fewer generations of peripheral airways may have contributed to the relatively normal LCI observed in EP children at 11 yrs of age and this could potentially have masked some ventilation inhomogeneity. However, it is equally possible that following BPD there may be relatively homogenous airway narrowing and hence minimal impact on the LCI. Whatever the underlying mechanism, it appears that the LCI would not be the method of choice when trying to identify airway abnormalities in children born EP, unless used in conjunction with spirometry. The reduced diffusion capacity amongst those born EP in this study has been previously reported [6] and probably reflects disruption of alveolar development (larger but fewer alveoli), decreased surface area for gas exchange and disrupted angiogenesis [33]. Nevertheless, very few EP children demonstrated evidence of restrictive lung disease, even when using a liberal definition based on both reduced FVC with normal FEV1/FVC. Of the 37 (76\%) EP children with technically acceptable plethysmographic lung volumes, only one (3\%) had evidence of restriction. In contrast, evidence of obstructive airway disease was observed in $45 \%$ of these children, $91 \%$ of whom were correctly identified by spirometry alone. Although it is recognised that plethysmography should be used to confirm restrictive lung disease, this technique may be difficult for young children especially if there is any developmental delay. Therefore, we adopted the more liberal definition including spirometry to ensure we did not underestimate any restriction in this population.

Indeed, although the aetiology of BPD has changed during the past four decades, a similar degree of respiratory dysfunction continues to be observed, albeit in increasingly immature survivors [2]. The increase in sReff among children with prior BPD, but not in those without, may be associated with increased bronchial smooth muscle and airway narrowing associated with the more prolonged and intense ventilatory support received by these children [34]. These findings of functional impairments are consistent with morphometric data from survivors with BPD [35].

The question of whether respiratory dysfunction following preterm birth is: 1) due to disruption of normal lung development 


\begin{tabular}{|c|c|c|c|c|}
\hline \multirow[t]{2}{*}{ TABLE 6} & en spirometric outc & nd specific airway & ce with extremely & birth and other \\
\hline & $\mathrm{zFEV}_{1}$ & zFEF $25-75 \%$ & zFEV1/FVC & ZsReff \\
\hline Subjects $\mathrm{n}$ & 94 & 94 & 94 & 100 \\
\hline Variability explained ${ }^{\#} \%$ & 41 & 36 & 20 & 13 \\
\hline Constant & -0.11 & -0.43 & 0.28 & 0.34 \\
\hline Wheeze ever" & $-0.71(-1.16--0.26)^{\star \star}$ & $-0.84(-1.32--0.36)^{\star \star}$ & $-0.66(-1.09--0.22)^{\star \star}$ & $0.38(0.04-0.73)^{*}$ \\
\hline \multicolumn{5}{|l|}{ Other variables of interest ${ }^{+}$} \\
\hline BPD & $-0.47(-1.10--0.15)$ & $-0.38(-1.05-0.29)$ & $-0.08(-0.69-0.53)$ & $0.30(-0.20-0.81)$ \\
\hline White mother & $0.43(-0.19-1.05)$ & $N A^{\S}$ & $N A^{\S}$ & $N A^{\S}$ \\
\hline
\end{tabular}

Data presented as coefficient $(95 \% \mathrm{Cl})$, unless otherwise stated. FEV1: forced expiratory volume in $1 \mathrm{~s}$; FEF25-75\%: forced expiratory flow between $25-75 \%$ forced vital capacity (FVC); sReff: specific effective airway resistance; EP: extreme pre-term; BPD: bronchopulmonary dysplasia; NA: not applicable. ${ }^{*}$ : adjusted $\mathrm{r}^{2}$ derived from multiple linear regression using the whole dataset (EP + controls). " : most significantly associated with these lung function outcomes. ${ }^{+}$: not significantly associated with the lung function outcomes after adjusting for the above variables. ${ }^{\text {s: }}$ factor not included in the model as it was not significantly associated with the lung function variable. With the exception of sReff, once accounted for this variable, additional symptoms and current asthma did not add significantly to the models. Current asthma status was significantly associated with a further increase in sReff by an average of $0.48 \mathrm{Z}$-score $(95 \% \mathrm{Cl} 0.07-0.90)(p=0.02)$ while the contribution from the "wheeze: ever" variable was no longer significant (mean Z-score $(95 \% \mathrm{Cl}) 0.26(-0.10-0.61)$ ). Although the univariate associations of BPD with lung function and of ethnicity with FEV 1 (but not FEF25-75\%, FEV1/FVC or sReff) (supplementary table E1) were no longer significant during multiple linear regression, they are reported as additional variables of interest since they show identical trends to those found when analysing the entire cohort [11]. *: $p<0.05 ;{ }^{* *}: p<0.01 ;{ }^{* * *} p<0.0001$.

following premature exposure of an immature lung to extrauterine conditions; 2) related to factors contributing to pre-term delivery; or 3) subsequent to lung injury incurred during resuscitation and subsequent ventilatory support during the neonatal period remains controversial due to the close interaction of these factors. While there is clear evidence of disrupted lung development following pre-term birth per se [36, 37], both respiratory morbidity and reductions in LF were far more severe in EP children with prior BPD. Indeed, the minority of EP children who survived without BPD were generally functioning remarkably well at $11 \mathrm{yrs}$ of age [11]. The fact that these functional abnormalities have persisted into early adolescence among those born pre-term suggests that the damage observed during infancy [38] may be permanent $[2,3]$.

\section{Conclusions}

There is a high incidence of persistent LF abnormalities among EP children born in the mid-1990s, which is largely obstructive in nature and likely to have long-term implications for future lung health. Spirometry proved to be an effective means of detecting these persistent abnormalities in survivors of EP birth and BPD, although discrimination could be improved in laboratory-based assessments by including measures of specific resistance and/or ventilation inhomogeneity. To minimise the risk of early onset chronic obstructive lung disease in adulthood, efforts should be made to preserve existing lung reserves by encouraging these children to lead a healthy lifestyle with respect to diet, exercise and avoidance of smoking.

\section{SUPPORT STATEMENT}

This work was supported by the UK Medical Research Council and Smiths Medical plc (London, UK) and Wellcome Trust Value in People Award. Research at the Institute of Child Health and Great Ormond Street Hospital benefits from R\&D funding from the NHS Executive. This paper was presented, in part, at the 2008 American Thoracic Society Conference (Toronto, Canada).

\section{STATEMENT OF INTEREST}

None declared.

\section{ACKNOWLEDGEMENTS}

EPICure Investigators Group: K. Costeloe (London), E.S. Draper (Leicester), E.M. Hennessy (London), N. Marlow (Nottingham; Chief Investigator) and J. Stocks (London, all UK). Developmental Panel: J. Fawke (Nottingham), S. Thomas (Leeds) and V. Rowell (London; all paediatricians); S. Johnson, R. Smith and R. Trikic (all psychologists); H. Palmer (study administrator). The EPICure Study Group comprises of paediatricians from 276 maternity units across the UK and Ireland who provided the original patients to the study, whose invaluable help we acknowledge in the establishment of these studies. The Investigator group was responsible for the funding and the overall design of the studies at $11 \mathrm{yrs}$ of age. The patients were recruited by J. Fawke, S. Thomas and V. Rowell. We would particularly like to thank the children and families who participated in this study.

\section{REFERENCES}

1 Field DJ, Dorling JS, Manktelow BN, et al. Survival of extremely premature babies in a geographically defined population: prospective cohort study of 1994-9 compared with 2000-5. BMJ 2008; 336: 1221-1223

2 Baraldi E, Filippone M. Chronic lung disease after premature birth. N Engl J Med 2007; 357: 1946-1955.

3 Wong PM, Lees AN, Louw J, et al. Emphysema in young adult survivors of moderate to severe bronchopulmonary dysplasia. Eur Respir J 2008; 32: 321-328.

4 Coalson JJ. Pathology of bronchopulmonary dysplasia. Semin Perinatol 2006; 30: 179-184.

5 Smith LJ, Van Asperen PP, McKay KO, et al. Reduced exercise capacity in children born very preterm. Pediatrics 2008; 122: e287-e293.

6 Vrijlandt EJ, Gerritsen J, Boezen HM, et al. Lung function and exercise capacity in young adults born prematurely. Am J Respir Crit Care Med 2006; 173: 890-896. 
7 Young RP, Hopkins R, Eaton TE. Forced expiratory volume in one second: not just a lung function test but a marker of premature death from all causes. Eur Respir J 2007; 30: 616-622.

8 Stern DA, Morgan WJ, Wright AL, et al. Poor airway function in early infancy and lung function by age 22 years: a non-selective longitudinal cohort study. Lancet 2007; 370: 758-764.

9 Halvorsen T, Skadberg BT, Eide GE, et al. Characteristics of asthma and airway hyper-responsiveness after premature birth. Pediatr Allergy Immunol 2005; 16: 487-494.

10 Narang I, Rosenthal M, Cremonesini D, et al. Longitudinal evaluation of airway function 21 years after preterm birth. Am J Respir Crit Care Med 2008; 178: 74-80.

11 Fawke J, Lum S, Kirkby J, et al. Lung function and respiratory symptoms at 11 years in extremely preterm children: the EPICure Study. Am J Respir Crit Care Med 2010; 182: 237-245.

12 Aurora P, Kozlowska WJ, Stocks J. Gas mixing efficiency from birth to adulthood measured by multiple-breath washout. Respir Physiol Neurobiol 2005; 148: 125-139.

13 Gustafsson PM, de Jong PA, Tiddens HA, et al. Multiple-breath inert gas washout and spirometry versus structural lung disease in cystic fibrosis. Thorax 2008; 63: 129-134.

14 Welsh L, Kirkby J, Lum S, et al. The EPICure study: maximal exercise and physical activity in school children born extremely preterm. Thorax 2010; 65: 165-172.

15 Tanner JM, ed. Growth at Adolescence. 2nd Edn. Oxford, Blackwell Scientific Publications, 1962.

16 Costeloe K, Hennessy E, Gibson AT, et al. The EPICure study: outcomes to discharge from hospital for infants born at the threshold of viability. Pediatrics 2000; 106: 659-671.

17 Kirkby J, Stanojevic S, Welsh L, et al. Reference equations for specific airway resistance in children: the asthma UK initiative. Eur Respir J 2010; 36: 622-629.

18 Rosenthal M, Cramer D, Bain SH, et al. Lung function in white children aged 4 to 19 years: II - single breath analysis and plethysmography. Thorax 1993; 48: 803-808.

19 Rosenthal M. Annual assessment spirometry, plethysmography, and gas transfer in cystic fibrosis: do they predict death or transplantation. Pediatr Pulmonol 2008; 43: 945-952.

20 Stanojevic S, Wade A, Cole TJ, et al. Spirometry centile charts for young Caucasian children: the Asthma UK Collaborative Initiative. Am J Respir Crit Care Med 2009; 180: 547-552.

21 Castile RG. Pulmonary function testing in children. In: Chernick V, Boat TF, Wilmott RW, Bush A, eds. Kendig's Disorders of the Respiratory Tract in Children. Philadelphia, Elsevier, 2006: 168-185.

22 Cole TJ, Freeman JV, Preece MA. British 1990 growth reference centiles for weight, height, body mass index and head circumference fitted by maximum penalized likelihood. Stat Med 1998; 17: 407-429.
23 Anderson HR, Gupta R, Strachan DP, et al. 50 years of asthma: UK trends from 1955 to 2004. Thorax 2007; 62: 85-90.

24 Stanojevic S, Wade A, Stocks J, et al. Reference ranges for spirometry across all ages: a new approach. Am J Respir Crit Care Med 2008; 177: 253-260.

25 Walker SM, Franck LS, Fitzgerald M, et al. Long-term impact of neonatal intensive care and surgery on somatosensory perception in children born extremely preterm. Pain 2009; 141: 79-87.

26 Baraldi E, Bonetto G, Zacchello F, et al. Low exhaled nitric oxide in school-age children with bronchopulmonary dysplasia and airflow limitation. Am J Respir Crit Care Med 2005; 171: 68-72.

27 O'Reilly MA, Marr SH, Yee M, et al. Neonatal hyperoxia enhances the inflammatory response in adult mice infected with influenza A virus. Am J Respir Crit Care Med 2008; 177: 1103-1110.

28 Schittny JC, Mund SI, Stampanoni M. Evidence and structural mechanism for late lung alveolarization. Am J Physiol Lung Cell Mol Physiol 2008; 294: L246-L254.

29 Thibeault DW, Mabry SM, Ekekezie II, et al. Collagen scaffolding during development and its deformation with chronic lung disease. Pediatrics 2003; 111: 766-776.

30 Lum S, Gustafsson P, Ljungberg H, et al. Early detection of cystic fibrosis lung disease: multiple-breath washout versus raised volume tests. Thorax 2007; 62: 341-347.

31 Hulskamp G, Lum S, Stocks J, et al. Association of prematurity, lung disease and body size with lung volume and ventilation inhomogeneity in unsedated infants: a multicentre study. Thorax 2009; 64: 240-245.

32 Latzin $\mathrm{P}$, Roth $\mathrm{S}$, Thamrin C, et al. Lung volume, breathing pattern and ventilation inhomogeneity in preterm and term infants. PLoS One 2009; 4: e4635.

33 Thebaud B, Abman SH. Bronchopulmonary dysplasia: where have all the vessels gone? Roles of angiogenic growth factors in chronic lung disease. Am J Respir Crit Care Med 2007; 175: 978-985.

34 Sward-Comunelli SL, Mabry SM, Truog WE, et al. Airway muscle in preterm infants: changes during development. J Pediatr 1997; 130: 570-576.

35 Howling SJ, Northway WH Jr, Hansell DM, et al. Pulmonary sequelae of bronchopulmonary dysplasia survivors: high-resolution CT findings. Am J Roentgenol 2000; 174: 1323-1326.

36 Friedrich L, Pitrez PM, Stein RT, et al. Growth rate of lung function in healthy preterm infants. Am J Respir Crit Care Med 2007; 176: 1269-1273.

37 Hoo AF, Dezateux C, Henschen M, et al. Development of airway function in infancy after preterm delivery. J Pediatr 2002; 141: 652-658.

38 Lum S, Hulskamp G, Merkus P, et al. Lung function tests in neonates and infants with chronic lung disease: forced expiratory maneuvers. Pediatr Pulmonol 2006; 41: 199-214. 